Z. Klin. Chem. Klin. Biochem.

11. Jg. 1973, S. 205-208

\title{
Lagerungsbedingte Fehler bei Creatinin-Bestimmungen
}

\author{
Von E. KIrberger ${ }^{1}$ ) und $H . K_{\text {ELLER }}$ \\ Klinisch-chemisches Zentrallaboratorium des Kantons St. Gallen
}

(Eingegangen am 20. Dezember 1972/23. Februar 1973)

\begin{abstract}
Heparin-Blutproben, geronnene Vollblut-Proben, Heparin-Plasma und Serum wurden daraufhin untersucht, ob Lagerung bei Raumtemperatur Einfluß auf die zu messenden Creatinin-Konzentrationen hat. Es zeigte sich, daß konventionelle Verfahren zunehmend falschhohe Creatinin-Werte liefern, wenn die Lagerungszeit länger als $12 \mathrm{~h}$ beträgt. Dieser Effekt ist bei Blutproben stärker ausgeprägt als bei Serum oder Plasma, kann aber auch hier statistisch sicher beobachtet werden. Dagegen liefert die Adsorption des Creatinins an Fullererde und nachfolgende Bestimmung mittels alkalischer Pikrinsäure auch bei längerer Lagerung unveränderte Werte. Daraus geht hervor, daß der lagerungsbedingte Fehler durch unspezifische Chromogene zustande kommt. Die Schlußfolgerungen, die sich für die Praxis aus diesen Beobachtungen ergeben, werden diskutiert.
\end{abstract}

\section{Errors in the determination of creatinine caused by storage}

The effect of storage at room temperature on the determination of creatinine was investigated in heparinized blood, coagulated whole blood, heparinized plasma and serum. It was found that conventional methods gave falsely high creatinine values after storage of the samples for more than $12 \mathrm{~h}$, and these became progressively higher with the length of storage. The effect is more marked in blood samples than in serum or plasma, but it is still statistically significant in the latter. On the other hand, the creatinine values were unchanged, even after extended storage, if the creatinine was adsorbed into Fuller's earth and then determined with the aid of alkaline picric acid. Thus the error due to storage is caused by unspecific chromogens. The significance of these observations in the clinical determination of creatinine is discussed.

Im Untersuchungsmaterial der Einsendepraxis beobachtete der eine von uns (E. K.) unerwartet häufig nicht korrelierende Harnstoff- und Creatinin-Werte. Systematisches Verfolgen dieser Beobachtung ließ vermuten, daß die erhöhten Creatinin-Werte dann auftraten, wenn das Untersuchungsmaterial längere Zeit gelagert worden war. Es wurde daher der Einfluß der Proben-Verwahrzeit auf die Ergebnisse der CreatininBestimmungen in geronnenen Vollblutproben oderSerum oder Plasma untersucht.

\section{Analytische Methoden}

A. Im Facharzt-Laboratorium in Frankfurt

1. Manuelle Creatinin-Bestimmung nach Popper et al. (1), benutzt wurden die Reagenzien det Biochemica Testkombination "Creatinin" (TC-CR 15943 TC AD) der Firma Boehringer Mannheim $\mathrm{GmbH}$.

2. Venöse Blutentnahmen in konventioneller Weise (Rekordspritze, Zentrifugen-Röhrchen aus Glas): nach eingetretener Gerinnung wurde das Blut sofort oder nach einer Lagerungsperiode abzentrifugiert und Creatinin im Serum bestimmt.

B. Im Hospital-Laboratorium, St. Gallen

1. Maschinelle Creatinin-Bestimmung: Technicon SMA 12/60, Methode nach Stevens et al. (2). Mit der Maschine werden die folgenden 12 Parameter simultan aus jeder Probe bestimmt: Natrium, Kalium, Chlorid, $\mathrm{CO}_{2}$, Calcium, Phosphat, TotalProtein, Cholesterin, Harnstoff, Creatinin, Harnsäure und Bilirubin.

2. Bestimmung des "wahren Creatinin" nach MüLLER et al. (3).

3. Bestimmung des Pyruvat nach Czok et al. (4).
4. Blutentnahmen mittels „Monovetten“ (5), die mit Ammoniumheparinat imprägniert waren. Mit Heparinat-Plasma wurde bei den hospitalisierten Patienten und bei den Probanden des Spitallaboratoriums gearbeitet. Das eingesandte Material ist auf konventionelle Weise gewonnen.

5. Statistische Auswertungen (Varianzanalyse, t-Test) wurden entsprechend CAMPBELL (6) durchgeführt.

\section{Ergebnisse}

Auswertung vorliegenden Zahlenmaterials des Spitallabors

a) Aus den Tageslisten wurden 200 Creatinin-Werte von internen Patienten ausgewählt, bei denen aufgrund der übrigen Befunde (Elektrolyte, Harnstoff-N) angenommen werden konnte, daß es sich um nierengesunde Patienten handelt: Kollektiv A der Abbildung 1. Das Zeitintervall zwischen Blutentnahme und Abzentrifugieren dürfte nicht länger als $60 \mathrm{~min}$ und zwischen Zentrifugieren und Analyse nicht mehr als $120 \mathrm{~min}$ betragen haben.

b) Ein analoges Zahlen-Kollektiv B wurde zusammengestellt aus Werten, die von geronnenen Vollblutproben externer Patienten stammten, vorwiegend aus benachbarten Spitälern eingesandt.

c) Ein weiteres analoges Kollektiv C wurde gebildet aus eingesandten Serum-Proben.

1) Dr. ERnSt Krrberger starb am 19. Oktober 1972, während der Fertigstellung dieser Arbeit. 

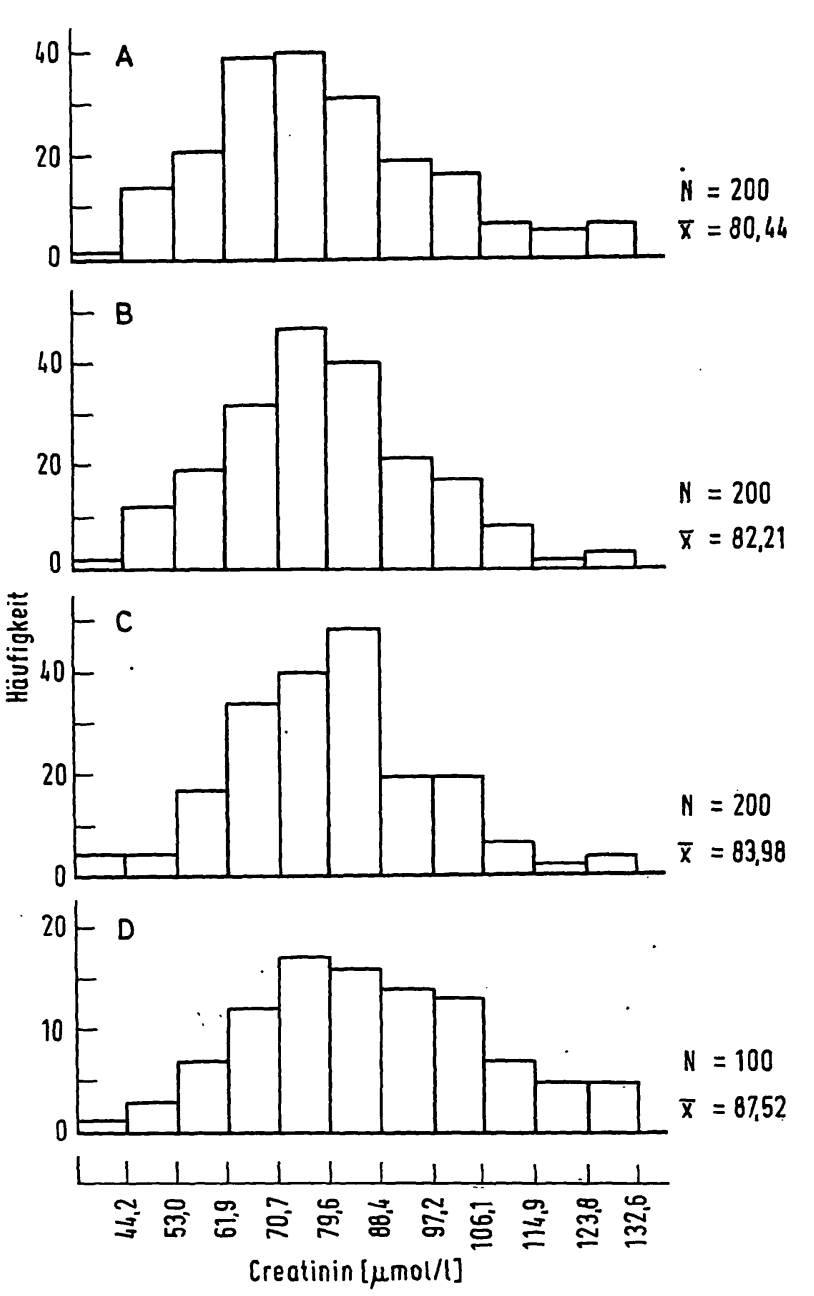

Abb. 1

Histogramme von Creatinin-Werten nierengesunder Patienten A. Hospitalisierte Patienten. Differenz zwischen Blutabnahme und Analyse weniger als $3 \mathrm{~h}$

B. wie A, jedoch eingesandtes Material in Form geronnener Blutproben C. wie A, jedoch eingesandtes Material in Form von Serum D. wie A, jedoch Material, das am Samstagnachmittag eingesandt wurde, und dessen größter Teil vermutlich länger als $48 \mathrm{~h}$ gelagert ist. Bei A, B, C Zahl der Probanden $=200$, bei D Zahl der Probanden $=100$

Tab. 1

Varianz-Analyse der Histogramme von Abbildung 1

\begin{tabular}{lcl} 
& F & \\
\hline$A \longrightarrow$ B & 8,88 & auf dem 1\% Niveau signifikant different \\
$\mathrm{A} \longrightarrow$ C & 7,38 & auf dem 1\% Niveau signifikant different \\
$\mathrm{A} \longrightarrow \mathrm{D}$ & 18,6 & auf dem $0,1 \%$ Niveau signifikant different \\
$\mathrm{B} \longrightarrow \mathrm{C}$ & 0,06 & nicht signifikant different \\
$\mathrm{B} \longrightarrow \mathrm{D}$ & 3,63 & nicht signifikant different \\
$\mathrm{C} \longrightarrow \mathrm{D}$ & 4,35 & auf dem 5\% Niveau signifikant different \\
\hline
\end{tabular}

d) Die Werte des Kollektivs $\mathrm{D}$ stammen von eingesandtem Untersuchungsmaterial, das jeweils am Montag analysiert worden war. Der größte Teil dieses Materials dürfte am Freitag entnommen worden sein, so $\mathrm{da} \beta$ dieses Material von der Blutentnahme bis zur chemischen Analyse länger als $48 \mathrm{~h}$ gelagert hat.

Die vorliegenden Kollektive wurden einer einfaktoriellen Varianz-Analyse unterworfen: Aus der $\mathrm{Ta}$ - belle 1 geht eindeutig hervor, daß frisch entnommenes Material (A) signifikant niedrigere Creatinin-Werte lieferte als die länger verwahrten Kollektive.

Beobachtung der Creatinin-Werte in Serum-, Plasma- und Blut-Proben während einer 5tägigen Lagerungsperiode des Untersuchungsmaterials, wobei zur Creatinin-Bestimmung konventionelle analytische Verfahren herangezogen wurden:

Von 12 gesunden Probanden wurden Blutproben unter Zusatz von Ammonium-Heparinat entnommen. Die Hälfte des Materials von jedem Patienten wurde sofort zentrifugiert und das überstehende Plasma in 4 Glasröhrchen (jeweils für einen Untersuchungstag) aufgeteilt. Die zwieite Hälfte wurde nicht zentrifugiert, sondern direkt jeweils in 4 Glasröhrchen abgefüllt. Die Glasgefäße wurden durch einen Schraubverschluß luftdicht verschlossen. Am Entnahmetag und den nächsten 4 folgenden Tagen wurde in den 12 Plasmaund in den 12 Blut-Specimen das Creatinin mit dem SMA $12 / 60$ bestimmt. In der Zwischenzeit lagerten die Proben bei $25^{\circ} \mathrm{C}$, wobei die Röhrchen horizontal lagen. Sie wurden während der Lagerungsperiode nicht bewegt.

Das Ergebnis dieser Untersuchungsserie zeigt Abbildung 2. Zur Prüfung, ob die Differenzen im Sinne einer Zunahme der "Creatinin"-Konzentrationen signifikant sind, wurde jeweils der t-Test für gepaarte Stichproben durchgeführt, die Resultate sind in der Tabelle 2 wiedergegeben.

Parallele Untersuchungen wurden im Facharzt-Laboratorium an geronnenen Blut- und Serum-Proben durchgeführt. Die manuelle Creatinin-Bestimmung. führte zu

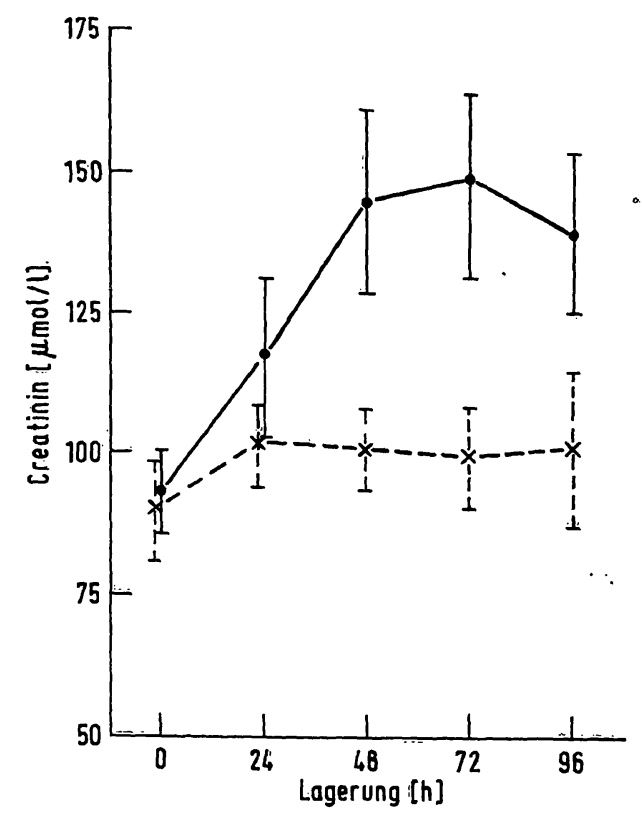

Abb. 2

Anderung der Creatinin-Werte von Hepärin-Blut/Plasma-Proben (12 nierengesunde Probanden) bei Lagerung bis zu $96 \mathrm{~h}$ Aufgetragen sind die Mittelwerte mit Streuungen $(1 \mathrm{~s})$ von gelagerten

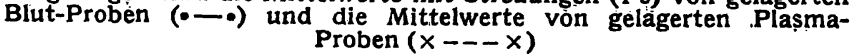


Tab. 2

t-Test für gepaarte Beobachtungen entsprechend Abbildung 2 Blut

\begin{tabular}{cllc}
\hline $\begin{array}{c}\text { Beobachtete } \\
\begin{array}{c}\text { Zeitspanne } \\
{[\mathrm{h}]}\end{array}\end{array}$ & $\begin{array}{c}\mathrm{N}=12 \\
\mathrm{FG}=11\end{array}$ & \\
\hline $0 \longrightarrow 24$ & $t=8,617$ & $\mathrm{p}<0,001$ & $99,9 \%$ \\
$24 \longrightarrow 48$ & $t=7,3127$ & $\mathrm{p}<0,001$ & $99,9 \%$ \\
$48 \longrightarrow 72$ & $t=1,678$ & $p<0,1$ & $90 \%$ \\
$72 \longrightarrow 96$ & $t=0,1815$ & $p<0,8$ & $\begin{array}{c}\text { keine signifikante } \\
\text { Differenz }\end{array}$ \\
\hline
\end{tabular}

\begin{tabular}{|c|c|c|c|}
\hline \multicolumn{4}{|c|}{ Plasma } \\
\hline $\begin{array}{c}\text { Beobachtète } \\
\text { Zeitspanne } \\
\text { [h] }\end{array}$ & & & $\cdot$ \\
\hline $\begin{array}{r}0 \longrightarrow 24 \\
24 \longrightarrow 48\end{array}$ & $\begin{array}{r}t=8,4177 \\
t=1,4755\end{array}$ & $\begin{array}{l}p<0,001 \\
p<0,2\end{array}$ & $\begin{array}{c}80 \% \\
\text { (fragliche Differenz) }\end{array}$ \\
\hline $\begin{array}{l}48 \longrightarrow 72 \\
72 \longrightarrow 96\end{array}$ & $\left.\begin{array}{l}t= \\
t=\end{array}\right\}$ keine & ikante Di & renz \\
\hline
\end{tabular}

qualitativ und quantitativ gleichen Resultaten: im geronnenen Vollblut ist in den ersten $48 \mathrm{~h}$ (bei Lagerung bei Raumtemperatur) ausnahmslos eine Zunahme der Creatinin-Konzentrationen von 3 bis $10 \mathrm{mg} / \mathrm{l} \mathrm{zu}$ beobachten. In Serum-Proben ist die Zunahme geringer, beschränkt sich auf die ersten $24 \mathrm{~h}$ und ist auch nicht regelmäßig in jeder Probe zu beobachten.

Vergleich konventioneller Creatinin-Bestimmungsverfahren mit Methoden zur Bestimmung des ;,wahren Creatinin"

Analog dem vorstehend geschilderten Vorgehen wurde in gelagerten Heparin-Blutproben von 11 gesunden Probanden das "wahre Creatinin" bestimmt. Als Vergleichsverfahren diente wiederum die Bestimmung

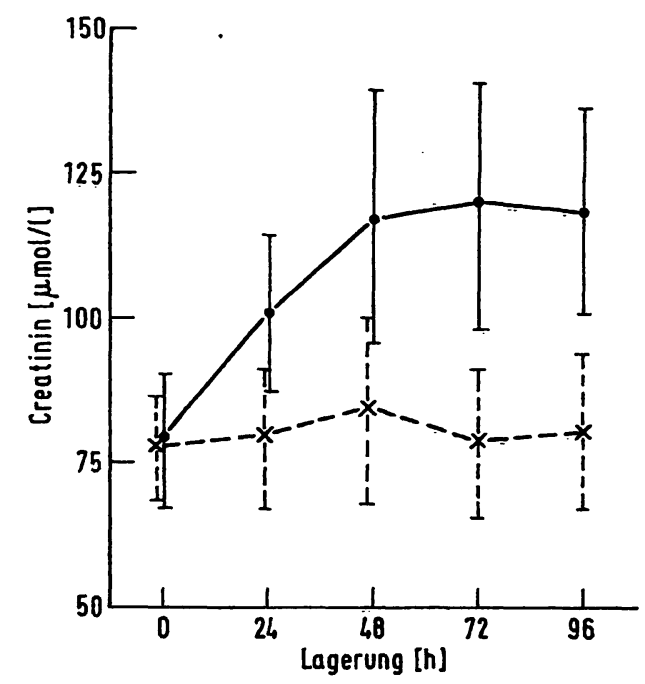

Abb. 3

Anderung der Creatinin-Werte von Heparin-Blut-Proben (11 nierengesunde Probanden) bei Lagerung bis $\mathrm{zu} 96 \mathrm{~h}$. Aufgetragen sind die Mittelwerte und Streuungen (1 s) der mit dem SMA $12 / 60$ bestimmten
Werte $(\bullet-\bullet)$ und der "wahren Creatinin-Werte" $(x--x)$ mit dem SMA 12/60. Die Abbildung 3 zeigt, daß mit dem Autoanalyzer eine (weitstreuende) scheinbare Vermehrung des Creatinin zu messen ist, während das "wahre Creatinin“ über die gesamte Versuchszeit unverändert bleibt.

\section{Besprechung der Ergebnisse}

Die scheinbare Zunahme des Creatinin in Heparin-Blut, geronnenen Vollblut-Proben, aber auch in Plasma und Serum während einer Lagerungsperiode ist offenbar durch unspezifische Chromogene (Übersicht bei l. c. $(7,8,9))$ bewirkt.

Orientierende Pyruvat-Bestimmungen (4) zeigten, daß die rasch einsetzende Pyruvat-Bildung in Vollblutproben allein nicht die Grundlage des beobachteten Effektes sein kann.

Durch Zwischenschalten eines adsorptiven Schrittes - Adsorption des Creatinin an Lloyds Reagenz (3, 10, 11) - können die auftretenden Störsubstanzen eliminiert werden. $\mathrm{Ob}$ auch andere Separationsverfahren, z. B. die Tetraphenylborat-Methode (8) oder die Oxidation der Störsubstanzen (Übersicht bei 1. c. $(7,11)$ ) ebenfalls ausreichend sind, und ob die kürzlich beschriebenen kinetischen Verfahren zur Bestimmung des Creatinin (12-15) den Lagerungsfehler umgehen, bleibt noch zu prüfen. Anzustreben ist eine spezifische, enzymatische Bestimmungsmethode (16-19).

Solange aber die unspezifische und unsensible JAFFÉReaktion in den heute üblichen Modifikationen ohne Berücksichtigung von Störsubstanzen eingesetzt wird, ergeben sich als Schlußfolgerungen aus den vorliegenden Befunden die folgenden Forderungen:

1. Werden zur Creatinin-Bestimmung Routine-Methoden vom Typ des Popper-Verfahrens eingesetzt, so darf die Proben-Verwahrzeit $5 \mathrm{~h}$ nicht überschreiten, weil danach der Einfluß des in der Probe entstehenden Pyruvats und eventuell anderer Störsubstanzen die Creatinin-Bestimmung meßbar beeinflussen kann.

2. Läßt sich diese Bedingung nicht einhalten, so muß Serum oder Plasma möglichst kurzfristig nach der Blutentnahme von den Erythrocyten getrennt und im Kühlschrank aufbewahrt werden. Zum Versand von Proben, in denen Creatinin bestimmt werden soll, ist nur Serum oder Plasma zulässig, eingesandtes Vollblut darf von einem qualifizierten Laboratorium nicht auf Creatinin untersucht werden.

3. Aber auch bei eingesandtem Serum oder Plasma ist bei der ärztlichen Interpretation von Creatinin-Werten zu berücksichtigen, daß es sich um eine Verfälschung nach oben handeln kann. Leicht erhöhte CreatininWerte müssen nicht unbedingt Ausdruck einer verminderten Nieren-Leistung sein. Eine rechnerische Korrektur der Creatinin-Werte ist nicht möglich, da die Abweichungen vom richtigen Ergebnis nicht voraussehbaren Schwankungen unterliegen, wie es $z$. B. auch für Kalium und Phosphat gilt. 


\section{Literatur}

$\therefore \because$

1. Popper, H., MANDel, E. \& Mayer, H. (1937), Biochem. Zeitschr. 291, 354 - 367. - 2. Stevkens, D. L., SkEkgs, L. T., Chasson, A. L., Gradx, H. T. \& Stanley, M: A: (1971), in "Reagent Data Handbook for the Technicon SMA 12/60 System" Technical Publication, No. THO-0160-10. - 3. MüLLER, H., Kluthe, R. \& AlexandRopouros, A. (1970), diese Z. 8, 394-397. - 4. Czok, R. \& Lamprecht, W. (1970), in Methoden der enzymatischen Analyse (Bergmeyer, H. U., Hrsg.), 2. Auf. Bd. II, S. 1407-1411, Verlag Chemie. - 5. KeLLER, H. (1971), Diagnostik 4, 23. - 6. CAMPBELL, R. C. (1971), Statistische Methoden für Biologie und Medizin, Thieme Verlag, Stuttgatt. - 7. HeNRY, R. J. (1964), Clinical Chemistry: Principles and Technics, Hoeber Medical Divis., New York. - 8. BexermanN, K. (1967), Clin. Chim. Acta 17, 47-52. - 9. Dreux, A. C. (1972), in "Quality
Control in Clin. Chemistry" (RAPPORT, A. E., ed.), A. Huber Publ. Bern, Stuttgart, Wien (1972). - 10. Borsook, H. (1935), J. biol. Chemistry 110, 481. - 11. Knoli, E. \& Stamm, D. (1970). diese Z. 8, 582. - 12. Barters, H. \& Bökmere, H. (1971), Clin. Chim. Acta 32, 81-85. - 13. Cook, J. G. H. (1971), Clin. Chim. Acta 32, 485-486. - 14. BlenNemann, H. (1971), Arztl. Lab. 17, 363-368. - 15. Bartels, H., BöHMER, M. \& HeIERLI, C. (1972), Clin. Chim. Acta 37, 193-197. - 16. DuBos, R. \& MrLLER, B. F. (1937), J. Biol. Chem. 121, 429-445. - 17. MrLLẼ, $\div$ B. F. \& Dubos, R. (1937), J. Biol. Chem. 121, 447-456. - 18. MilLER, B. F. \& Dusos, R. (1937), J. Biol. Chem. 121, 457-464. 19. WAHLEFELd, A. W., Herz, G. \& BeRGMEYER, H. U. (1972). Scand. J. Clin. Lab. Invest. 29 Suppl. 126.

Prof. Dr. med et rer. nat. Herbert Keller Klinisch-chemisches Zentrallaboratorium Frohbergstraße 3

CH-9000 St. Gallen 